\title{
Measuring the Properties of an Information Model : The IM Meter
}

Stefan Wallin Erisoft AB

Stefan.Wallin@sa.erisoft.se

+4691051733

In almost every area in human life measurements are important tools for us to understand our surroundings better. We can for instance measure the speed of a car to see if it is driving to fast, or measure the amount of sugar when we are making a cake. In the same way it is important for us to measure the features of various information models, because it will give us the opportunity to distinguish which models that are more complicated than others. With this measure it is also possible to estimate for example, the lines of code in the implemented product and the cost and time effort needed to make the product. It is also possible to discover which parts of the model that are most complex and thereby know which part which that will need the most time to implement.

The IM Meter is able to measure the significant properties of various information models such as complexity and information content. The measures the IM Meter deals with are of various sorts. Some of them measure the features of minor details of the information models while other measure more general and extending properties.

Since no one really knows what makes an information model complex, we decided that we should give the user so much valuable information as possible. The user than can decide by himself which measure he wants to use. There is also some values that can be used to see what part of an information model that is most complicated. You can also take some information models and see which one of them that are most complicated. These measures are calculated as weighted sums of the form $\lambda_{1} X_{1} \ldots \lambda_{n} X_{n}$ where $X_{1} \ldots X_{n}$ are the $n$ different quantities describing the IM, which are to be weighted together with the weighting parameters $\lambda_{1} \ldots \lambda_{n}$. The user of the IMMeter is allowed to change the weighting parameters so that he can give the parts of the IM that he think is difficult higher values.

The main window of the IM Meter is shown below..

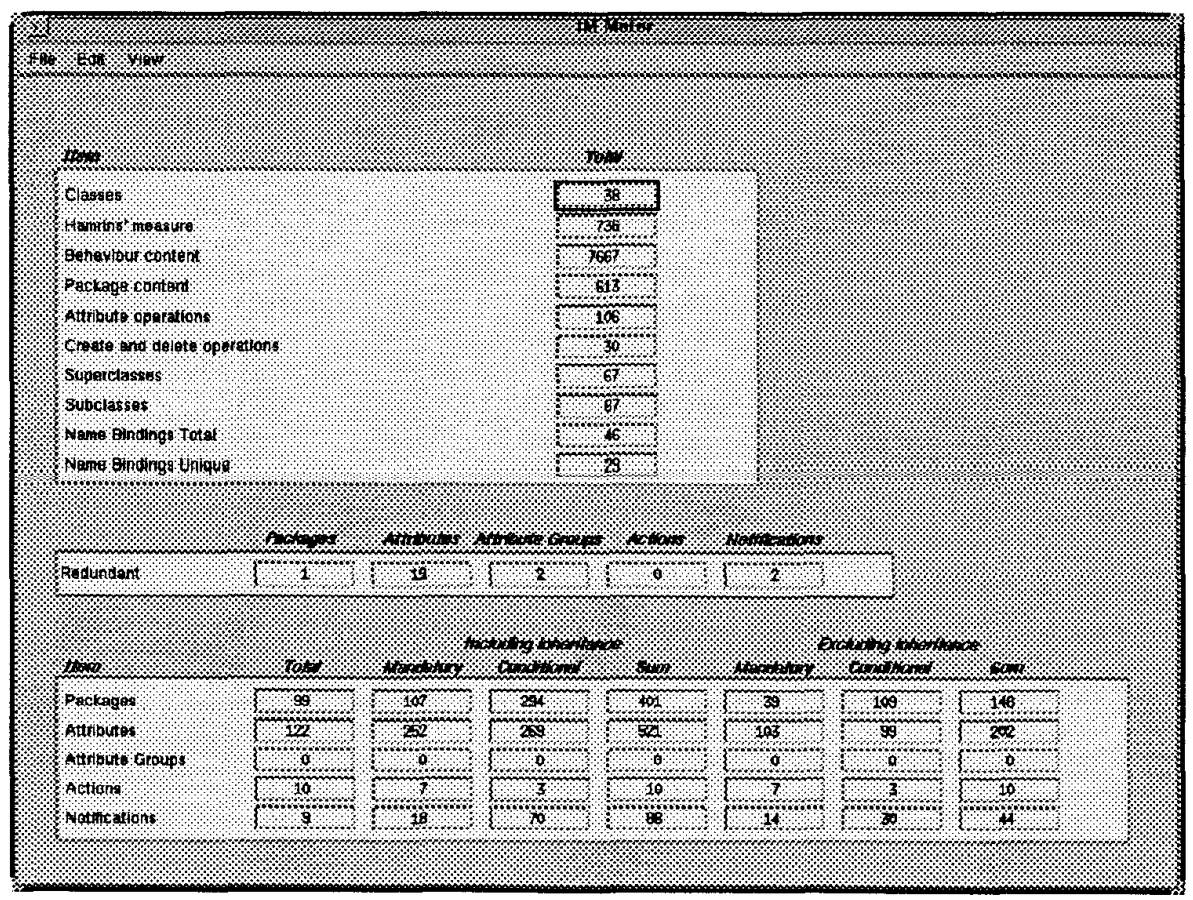


MEASURING

THE

PROPERTIES

OF AN

INFORMATION MODEL

o Everyone talks about the complexity of TMN but noone measures it.

1 (6) Stefan Wallin Erisoft AB

Measuring TMN Interface Complexity

\section{Why measure?}

\section{o Better understand our} surroundings:

When you can measure what you are speaking about, and express it into numbers, you know somothing about it; but when you cannot measure it, when you cannot express it in numbers, your knowledge is of a meagre and unsatisfactory kind: It may be the beginning of knowledge, but you have scarely in your thoughts advanced to the stage of science.

Lord Kelvin (1824-1924)

In almost every area in human life measurements are important tools for us to get to understand our surroundings better. For Instance, we measure the speed of a car to see if it is driving too fast and we count the number of students in the classroom to seo if anyone is missing... In the same manner It is important for us to be able to measure the features of various Information models, since this gives us the opportunity of distinguishing which models are more complicated than others. We can also estimate, for example, the cost, time and effort needed to implement various models, anc moreover discover which parts of the model are the most complex and thereby contribute the most to the implementIng costs. 


\section{IMMeter}

o A tool to perform complexity measurments of TMN Information Models written in GDMO/ASN.1

o Probe the properties of Information Models

complexity of the IM

Amount of information in the IM

S1ze and shape of the IM

o The measures are displayed in 6 windows

O ELEMENTARY MEASURES - the number of a specific item

EX. The number of classes in the IM

o COMPLEX MEASURES weighted sums of various different measures

3 (6) Stefan Wallin Erisoft AB

\section{Measuring TMN Interface Complexity}

\section{Complex measures}

o Since there are many characteristic features of an IM which together contribute to the complexity and the amount of information in the model, there are a need for more extensive measures taking many properties of the model into account. This is done in the IM Meter by means of weighted sums of the form:

$\lambda_{1} X_{1}+\ldots+\lambda_{n} X_{n}$

o $X_{i}=$ quantity describing the $\mathrm{IM}$, $i=1, \ldots, n$

o $\lambda_{i}=$ weight factor, $0 \leq \lambda_{i} \leq 1$,

o $\lambda_{j}=1 \Leftrightarrow$ the $j$ :th quantity $X_{i}$ is irrelevant to the weighted sum

o The user of the IM Meter has the possibility of defining the parameters manually or by using default values. 
Measuring TMN Interface Complexity

4 categories of complex measures

o Package content

o Attribute operations

o Create and delete operations

o Hamrin's measure

Describes the features of the entire IM

Consists of a collection of measures that are representative to an IM

In the IM Meter in particular it consists of 12 different measures

5 (ด) Sufan Wallin Erisoft AB

Measuring TMN Interface Complexity Hamrins measure vs Lines of code in implementation

c Classes from M.3100 and X.721

Table 1: The different classes in the crosh-connector
\begin{tabular}{|l|l|l|l|}
\hline \multicolumn{1}{|c|}{ MO Class } & Lines of code (I) & $\begin{array}{c}\text { Hamrin's } \\
\text { measure (b) }\end{array}$ & Vh \\
\hline \hline AlarmS.A.P & 705 & 74 & 9.5 \\
\hline CTP1 & 6310 & 748 & 8.5 \\
\hline $\begin{array}{l}\text { Fabric } \\
\text { CrossConn. }\end{array}$ & 8600 & 537 & 16.0 \\
\hline ME & 2160 & 214 & 10.0 \\
\hline AlarmRec. & 1180 & 111 & 10.6 \\
\hline AVCR & 591 & 104 & 5.7 \\
\hline $\begin{array}{l}\text { Discrim. } \\
\text { EFD }\end{array}$ & 1400 & 274 & 5.1 \\
\hline $\begin{array}{l}\text { LogRecord } \\
\text { EventLogR. }\end{array}$ & 1122 & 114 & 9.8 \\
\hline Log & 2000 & 157 & 12.7 \\
\hline
\end{tabular}

